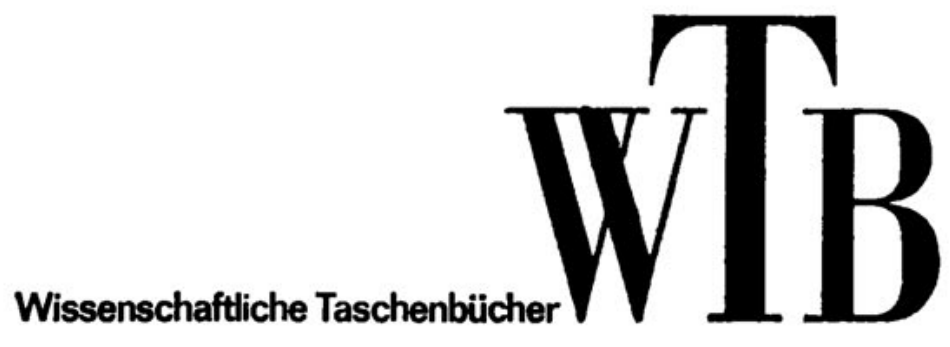

\title{
Chemie
}

Joachim Schuppan

Theorie

und

Meßmethoden

der

Konduktometrie 


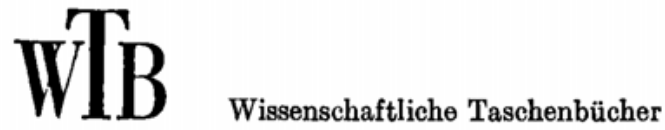

Eine Auswahl

lieferbarer Bände:

HEINRICH ARNOLD

Physikallsche Chemle der Halbleiter

Josohm AUth / Dretmar Grazow

KLAUS H. HERRMANN

Photoelektrische Erscheinungen

HANS BANDEMER

ANDREAS BELLMANN

WOLBHART JUNG / KLAUS RIOHTER

Optimale Versuchsplanung

Frank BeIOHeLt

Prophylaktlsche Erneuerung

von Systemen

JOACHIM BeLLACH / PETER Franken ELKE WARMUTH / WALTER WARMUTH $\mathrm{MaB}$, Integral

und bedingter Erwartungswert

\section{WOLFRAM BRAUER}

HANS-W ALDEMAR STRRITWOLF

Theoretische Grundlagen

der Halblelterphysilk

SIEOPRIED BREHMER

Elnfuhrung in die MaBtheorle

SIratried BrbHMER

Hilbert-Rsume und Spektralmaße

John Cunningham

Vektoren

GEORG DAUTOOURT

Relativistische Astrophyslk

K. Ch. Delokarov

Relatlvitststheorle und Materlallsmus

\author{
HANNELORE FISOHER \\ JOAOHTM PIEHLER \\ Modellsysteme \\ der Operationsforschung \\ Gotryried Fritzsohe \\ Grundlagen und Entwur? \\ passiver Analogzwelpole \\ Netzwerke I \\ Entwurf passiver Analogvierpole \\ Netzwerke II
}

HRRBERT GOERING

Asymptotlsche Methoden zur Losung von Differentialgleichungen

Herbert GoHring

Elementare Methoden zur Losung von Differentialglelchungsproblemen

Eduard Herlt / Nikolads Salik Spezlelle Relativitaitstheorle

HRLMUT HeSS

Der elektrische Durchschlag in Gasen

V. I. KARPMAN

Nichtllneare Wellen

In dispersiven Medien

ULRIOH KAUSMANN

KLAUS LOMMATZSOH

FrantTSEk Nožtcka

LIneare parametrische Optimlerung

A. R. KESsEL

Akustische Kernresonanz

KONRAD KRBHER

Festkörperphysik

DIETER KRESS

Theoretische Grundlagen der Signalund Informatlonsubertragung 